\title{
EDITORIAL
}

\section{Beyond the speed of SOUND}

\author{
M. Ahmed ${ }^{1}$ (1)
}

Received: 22 March 2020 / Accepted: 22 June 2020 / Published online: 27 June 2020

(c) The Japanese Breast Cancer Society 2020

\begin{abstract}
Much anticipation awaits the results of the SOUND trial, (Gentilini and Veronesi in Breast 21:678-681,2012) which may prove the futility of performing sentinel node biopsy (SNB) in low-risk breast cancer patients. However, do we really not know the answer to the questions that the SOUND trial poses already? Consideration must be taken of the very much overlooked trials predating the sentinel node era, which risk stratified patients according to the absence of palpable lymphadenopathy and without dependence upon ultrasound imaging (clinically negative axilla). This automatically selects a low-risk group of patients for axillary disease (low axillary burden) and the relevance of these critical trials is discussed.
\end{abstract}

Much anticipation awaits the results of the SOUND trial, [1] which may prove the futility of performing sentinel node biopsy (SNB) in low-risk breast cancer patients. This trial has randomized patients with early breast cancer (T1) and a negative preoperative assessment of the axilla on ultrasound to either sentinel node biopsy or observation alone, utilizing a primary endpoint of disease-free survival with a $2.5 \%$ non-inferiority margin. However, do we really not know the answer to the questions that the SOUND trial poses already? Consideration must be taken of the very much overlooked trials predating the sentinel node era, which risk stratified patients according to the absence of palpable lymphadenopathy and without dependence upon ultrasound imaging (clinically negative axilla). This automatically selects a low-risk group of patients for axillary disease (low axillary burden). These pioneering trials randomized this stratified group of patients between axillary node clearance and no axillary surgery. The most pertinent results can be derived from the trials, which did not involve adjuvant regional nodal irradiation. They provide an insight into the outcomes of omission of axillary surgery.

The NSABP-04 trial [2] — with over 25-years of followup-randomized breast cancer patients with clinically negative axillae to radical mastectomy and axillary node clearance, total mastectomy and regional irradiation or total

M. Ahmed

muneer.ahmed@ucl.ac.uk

1 Division of Surgery and Interventional Science, University College London, Royal Free Hospital, 9th Floor (East), Pond Street, London NW3 2QG, UK mastectomy alone. No difference in disease-free (DFS) or overall survival (OS) was identified in three-way comparison between any of the groups. The best local control was provided by the addition of regional radiation to mastectomy (1, 5 and $7 \%$ local recurrence for mastectomy and radiotherapy, radical mastectomy and no further axillary treatment, respectively). Of the patients undergoing radical mastectomy, $40 \%$ had histological confirmation of tumour positive nodes. Assuming that this would be representative of the axillary burden across all groups, it is important to highlight that the axillary recurrence rate was only $18.6 \%$ for total mastectomy alone-framed in the context that no patients received adjuvant systemic therapy in this trial. This demonstrates that half of all positive axillary disease is clinically occult even without systemic therapy.

Martelli et al. [3] randomized 238 patients aged 65-80 with T1N0 disease to breast-conserving surgery with or without axillary clearance. All patients received whole breast radiotherapy and tamoxifen. Cumulative incidence curves for breast cancer mortality at 15 years did not demonstrate any significant difference $(P=0.64)$. Of the 109 patients in the axillary clearance group, $23 \%$ had involved axillary nodes with no axillary recurrences. In the 110 patients in the no axillary clearance group, only four developed ipsilateral axillary disease. Due to slow recruitment in the older patient population, this trial terminated early and was succeeded by the INT09/98 trial, [4] which randomized 565 patients aged $18-65$ with T1N0 disease to either quadrantectomy with axillary clearance (QUAD arm) or quadrantectomy alone (QU arm). All patients received adjuvant whole breast radiotherapy with no attempts for 
regional nodal irradiation. Adjuvant medical therapies were determined upon the basis of receptor status, grade and nodal status in the axillary clearance arm only. After a median follow-up of 127.5 months, $8.5 \%$ of the QUAD arm and $8.2 \%$ of the QU group developed distant metastases $(P=1.000)$ and there were $6.3 \%$ and $6.2 \%$ deaths attributable to breast cancer in these groups, respectively $(P=1.000)$. In the QUAD arm 78 out of 272 patients $(28.7 \%$ ) had node positive disease and of these node positive patients, $11 \mathrm{had}$ greater than 3 involved nodes (high axillary burden-4\%). There were no axillary recurrences in the QUAD arm, but 22 out of 245 patients $(9.0 \%)$ in the QU arm. Half of these axillary recurrences $(4 \%)$ can be explained by the preoperatively undiagnosed high axillary burden - as identified on final histopathology in the QUAD arm. The other half, by the automatic use of chemotherapy in node positive patients in the QUAD arm, which is of course absent from the QU arm.

The IBCSG 10-93 trial, [5] randomized T1-3N0 breast cancer patients who were postmenopausal to either breast surgery with axillary node clearance followed by 5 years of tamoxifen or breast surgery alone followed by 5 years of tamoxifen. There was no difference in 6-year DFS $(\mathrm{HR}=1.06 ; 95 \% \mathrm{CI} 0.79-1.42 ; P=0.69)$ or $\mathrm{OS}(\mathrm{HR}=1.05$; $95 \%$ CI $0.76-1.46 ; P=0.77$ ) between the two groups. Of 234 patients in the axillary clearance arm, 68 were node positive. Of these, 18 had multiply involved nodes, recorded as greater than 4 . The axillary recurrence rate was only $1 \%$ versus $3 \%$ in the axillary clearance and breast surgery alone groups, respectively.

Avril et al. [6] randomized 625 patients, over 50 years of age with T1N0 disease to either breast surgery and axillary clearance $(\mathrm{Ax})$ or breast surgery alone (no-Ax). Unfortunately, the trial was statistically underpowered to demonstrate equivalence in OS between the groups. However, it yielded interesting results regarding axillary events-which were absent in the Ax group and recorded as only $2 \%$ in the no-Ax group. Even more interestingly, in the Ax arm, $14 \%$ of patients were node positive and assuming a similar distribution in the no-Ax arm, one would have expected the axillary event rate to be closer to this figure in that latter group. However, the fact that it was only $2 \%$, indicates that only a small fraction of axillary metastases become clinically relevant, the vast majority remaining occult.

The option of axillary radiotherapy was considered by Veronesi et al. [7] who randomized 435 patients with tumours up to $1.2 \mathrm{~cm}$ undergoing breast-conserving surgery to axillary radiotherapy or no axillary management. All patients received whole breast radiotherapy and adjuvant therapies according to their oestrogen receptor status. There was no significant difference in 5-year DFS between the two groups (HR $=1.59 ; 0.65-3.89)$ and at median follow-up of 63 months, three patients developed axillary metastases in the no radiotherapy arm and one within in.
These trials demonstrate that over half of all axillary metastases are clinically occult and do not warrant treatment [2]. Significant metastases can be identified in the modern era by the adjunct of axillary ultrasound compared to clinical palpation alone. The addition of systemic therapy and whole breast radiotherapy is able to half the axillary recurrence rate further compared to no further axillary management at all $[2,4]$ and the application of axillary radiotherapy is able to bring axillary recurrence to less than $1 \%[2,7]$. The selection of low-risk, post-menopausal candidates also offer excellent local control of under 3\% local recurrence [5].

When the points demonstrated by these trials-that predate sentinel node biopsy - are taken into consideration, it is clear that we already know what the answer to the SOUND trial will be. We have to concede that the evidence for deescalation of axillary surgical management is not based purely upon the results of Z11, [8] but rather an evolving body of work, which has emerged over the last 30 years. It is essential that this is embraced and implemented-rather than denied and obstructed-to ensure patients can benefit from reduced surgical morbidity.

\section{Compliance with ethical standards}

Conflict of interest The authors have no disclosures to make concerning financial and personal relationships with other people or organisations that could inappropriately influence their work. No funding was received and no ethical approval was requested for this work, as no studies with human participants or animals were performed by the author.

\section{References}

1. Gentilini O, Veronesi U. Abandoning sentinel lymph node biopsy in early breast cancer? A new trial in progress at the European institute of oncology of Milan (SOUND: Sentinel node vs. Observation after axillary UltraSouND). Breast. 2012;21(5):678-81.

2. Fisher B, Jeong JH, Anderson S, Bryant J, Fisher ER, Wolmark $\mathrm{N}$. Twenty-five-year follow-up of a randomized trial comparing radical mastectomy, total mastectomy, and total mastectomy followed by irradiation. N Engl J Med. 2002;347(8):567-75.

3. Martelli G, Boracchi P, Ardoino I, Lozza L, Bohm S, Vetrella G, Agresti R. Axillary dissection versus no axillary dissection in older patients with T1N0 breast cancer: 15-year results of a randomized controlled trial. Ann Surg. 2012;256(6):920-4.

4. Agresti R, Martelli G, Sandri M, Tagliabue E, Carcangiu ML, Maugeri I, Pellitteri C, Ferraris C, Capri G, Moliterni A, Bianchi G, Mariani G, Trecate G, Lozza L, Langer M, Rampa M, Gennaro M, Greco M, Menard S, Pierotti MA. Axillary lymph node dissection versus no dissection in patients with T1N0 breast cancer: a randomized clinical trial (INT09/98). Cancer. 2014;120(6):885-93.

5. International Breast Cancer Study G, Rudenstam CM, Zahrieh D, Forbes JF, Crivellari D, Holmberg SB, Rey P, Dent D, Campbell I, Bernhard J, Price KN, Castiglione-Gertsch M, Goldhirsch 
A, Gelber RD, Coates AS. Randomized trial comparing axillary clearance versus no axillary clearance in older patients with breast cancer: first results of international breast cancer study group trial 10-93. J Clin Oncol. 2006;24(3):337-44.

6. Avril A, Le Bouedec G, Lorimier G, Classe JM, Tunon-de-Lara C, Giard S, MacGrogan G, Debled M, Mathoulin-Pelissier S, Mauriac L, Group A. Phase III randomized equivalence trial of early breast cancer treatments with or without axillary clearance in post-menopausal patients results after 5 years of follow-up. Eur J Surg Oncol. 2011;37(7):563-70.

7. Veronesi U, Orecchia R, Zurrida S, Galimberti V, Luini A, Veronesi P, Gatti G, D'Aiuto G, Cataliotti L, Paolucci R, Piccolo P, Massaioli N, Sismondi P, Rulli A, Lo Sardo F, Recalcati A, Terribile D, Acerbi A, Rotmensz N, Maisonneuve P. Avoiding axillary dissection in breast cancer surgery: a randomized trial to assess the role of axillary radiotherapy. Ann Oncol. 2005;16(3):383-8.

8. Giuliano AE, McCall L, Beitsch P, Whitworth PW, Blumencranz P, Leitch AM, Saha S, Hunt KK, Morrow M, Ballman K. Locoregional recurrence after sentinel lymph node dissection with or without axillary dissection in patients with sentinel lymph node metastases: the American college of surgeons oncology group Z0011 randomized trial. Ann Surg. 2010;252(3):426-32.

Publisher's Note Springer Nature remains neutral with regard to jurisdictional claims in published maps and institutional affiliations. 